\title{
The Galactic center: not an active galactic nucleus
}

\author{
Deokkeun An ${ }^{1}$, Solange V. Ramírez ${ }^{2}$ and Kris Sellgren ${ }^{3}$ \\ ${ }^{1}$ Dept. of Science Education, Ewha Womans University, \\ Seoul 120-750, Republic of Korea \\ email: deokkeun@ewha.ac.kr \\ ${ }^{2}$ NASA Exoplanet Science Institute, California Institute of Technology, \\ Mail Stop 100-22, Pasadena, CA 91125, USA \\ ${ }^{3}$ Dept. of Astronomy, Ohio State University, \\ 140 West 18th Avenue, Columbus, OH 43210, USA
}

\begin{abstract}
We present $10 \mu \mathrm{m}-35 \mu \mathrm{m}$ Spitzer spectra of the interstellar medium in the central molecular zone $(\mathrm{CMZ})$, the central $210 \mathrm{pc} \times 60 \mathrm{pc}$ of the Galactic center (GC). We present maps of the $\mathrm{CMZ}$ in ionic and $\mathrm{H}_{2}$ emission, covering a more extensive area than earlier spectroscopic surveys in this region. We compare diagnostic line ratios measured in the Spitzer Infrared Nearby Galaxies Survey to our data. Previous work shows that forbidden line ratios can distinguish starforming galaxies from low-ionization nuclear emission-line regions (LINERs) and active galactic nuclei (AGNs). Our GC line ratios agree with star-forming galaxies and not with LINERs or AGNs.
\end{abstract}

Keywords. galaxies: active, galaxies: ISM, galaxies: nuclei, galaxies: starburst, infrared: ISM, ISM: molecules, stars: formation

\section{Introduction}

A detailed spectroscopic study of the interstellar medium (ISM) over a wide range of wavelengths can provide important constraints on the process of star-forming activities in the central molecular zone (CMZ; Morris \& Serabyn 1996). Mid-IR emission lines are particularly useful because the CMZ gas lies behind heavy dust obscuration. Forbidden emission lines in the mid-IR are generally insensitive to electron gas temperatures, and can be used to determine physical properties such as the electron density and ionization parameters, and to identify sources of ionization.

In our recent spectroscopic survey of massive young stellar objects (YSOs) in the CMZ (An et al. 2009, 2011), we used Spitzer/IRS to collect an extensive set of mid-IR spectra for 107 YSO candidates. The goal of this survey was to discover and characterize the spectroscopic properties of massive YSOs in the Galactic center (GC). To achieve this original goal, we spent half of our observing time on background spectra near each YSO candidate because of the strong and spatially variable background emission in the GC. Our GC background spectra, which are the by-product of our YSO observing program, now constitute the largest and most comprehensive mid-IR spectroscopic data set available to study the properties of the ionized and molecular gas of the Galactic nucleus.

Here we report the observational evidence that the ISM in the CMZ is characterized by the mid-IR emission properties of star-forming regions as observed in nearby galaxies. A full description of the method and results is found in An et al. (2013). Here we only discuss the highlights. 


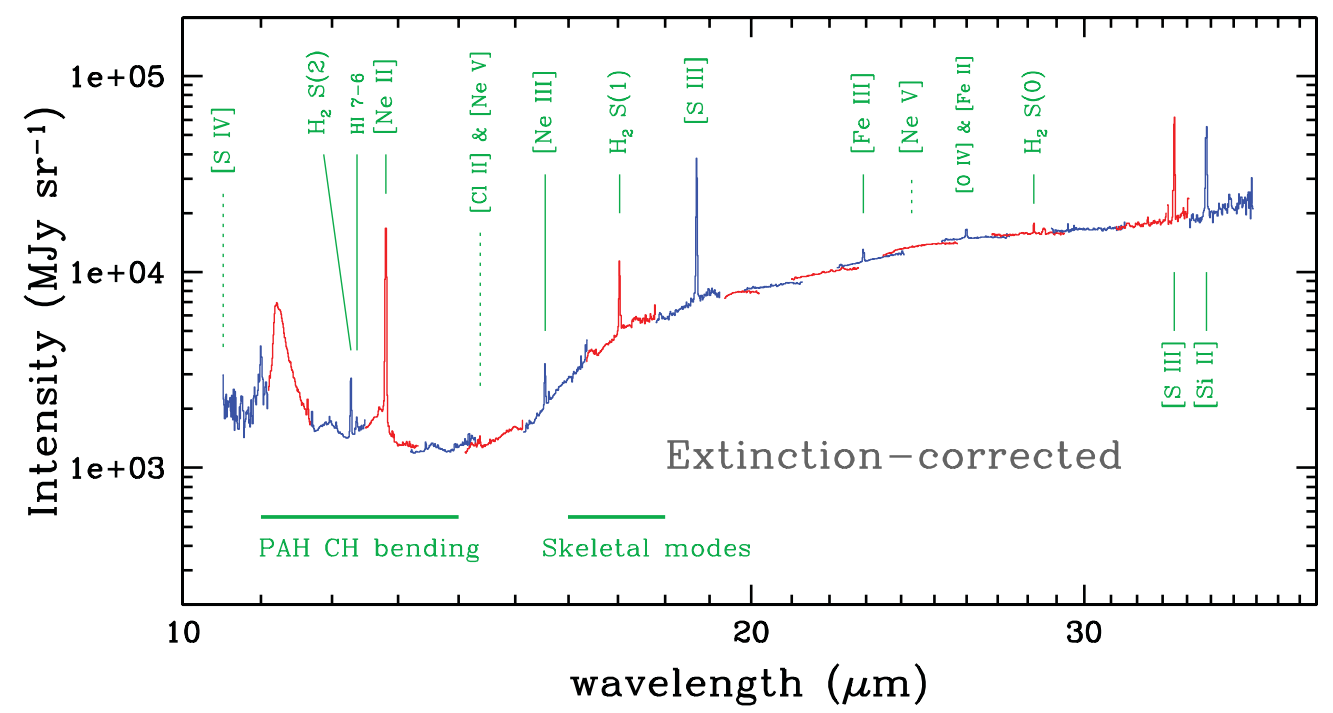

Figure 1. Coadded GC ISM spectra constructed from 428 high-resolution IRS spectra, after correcting individual spectra for extinction based on the ratio between $10 \mu \mathrm{m}$ and $14 \mu \mathrm{m}$ continuum fluxes (Simpson et al. 2007). Locations of ionic forbidden emission lines and molecular hydrogen lines are marked, where dotted lines indicate positions of weakly detected emission lines. [A COLOR VERSion is AVAILABLE ONLINE.]

\section{Mid-IR interstellar medium spectra}

In the analysis of the GC ISM, we used high-resolution Spitzer/IRS spectra obtained for 428 individual lines of sight in the CMZ. Detailed information on the data acquisition and reduction is found in An et al. (2009, 2011). We measured emission line fluxes from several ionic fine-structure lines and $\mathrm{H}_{2}$ lines by fitting a Gaussian. These line flux measurements were corrected for foreground extinction between the GC and the Sun on a spectrum-to-spectrum basis, because of the patchy dust extinction in the GC. This was done by comparing continuum flux ratios at $10 \mu \mathrm{m}$ and $14 \mu \mathrm{m}$ to a template value, as developed in Simpson et al. (2007).

In addition to individual ISM spectra, we also created and analyzed a coadded GC spectrum as shown in Figure 1. We constructed the coadded IRS spectrum by summing fluxes from 428 individual high-resolution spectra after foreground extinction correction. This allows us to directly compare line ratios measured across a $210 \mathrm{pc} \times 60 \mathrm{pc}$ region of the CMZ to the ratios of ionic lines measured in extragalactic sources in the Spitzer Infrared Nearby Galaxies Survey (SINGS; Dale et al. 2009). The SINGS galaxy sample (Kennicutt et al. 2003) has a median distance of $9.5 \mathrm{Mpc}$, and the closest is at $0.6 \mathrm{Mpc}$. The IRS high-resolution modules, $\mathrm{SH}$ and $\mathrm{LH}$, have a slit entrance size corresponding to $14 \mathrm{pc} \times 33 \mathrm{pc}$ and $32 \mathrm{pc} \times 65 \mathrm{pc}$, respectively, for a galaxy at $0.6 \mathrm{Mpc}$, or $220 \mathrm{pc} \times 520$ $\mathrm{pc}$ and $510 \mathrm{pc} \times 1000 \mathrm{pc}$ for a galaxy at $9.5 \mathrm{Mpc}$. Our coadded spectrum of the CMZ is a good match to the SINGS spatial resolution.

\section{Results}

Mapping results for forbidden line ratios, [SiII] $34.82 \mu \mathrm{m} /$ [SIII] $33.48 \mu \mathrm{m}$ and [OIV] $25.89 \mu \mathrm{m} /[\mathrm{NeII}] 12.81 \mu \mathrm{m}$, are displayed in Figure 2. Line ratios were computed from extinction-corrected line fluxes, when they were detected at more than a $3 \sigma$ level. Each pixel in the map covers a $1.5^{\prime} \times 1.5^{\prime}$ region of the sky $(\sim 3.5 \mathrm{pc} \times 3.5 \mathrm{pc})$, which is an 

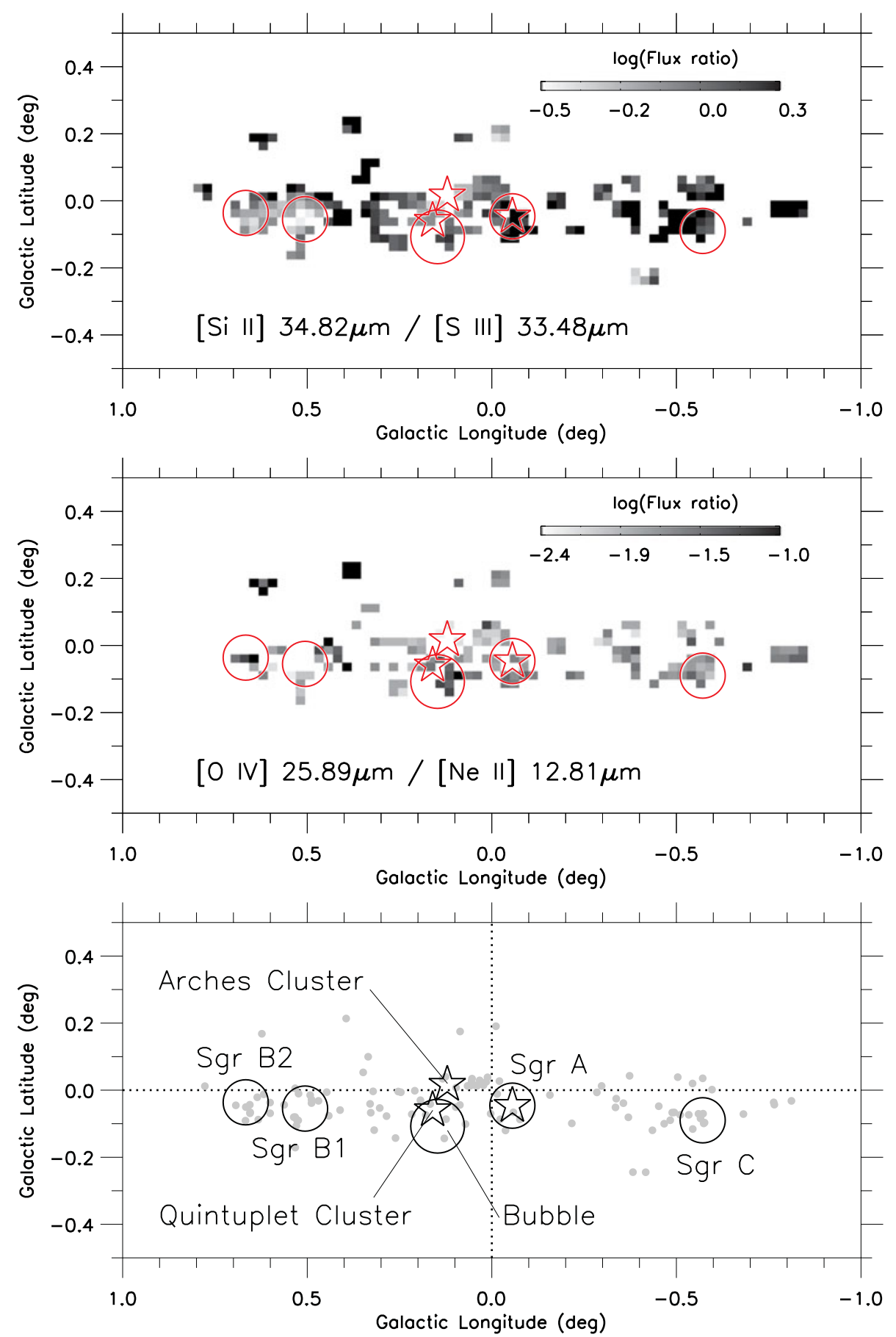

Figure 2. Panoramic maps of line intensity ratios in the GC, displaying [SiII] $34.82 \mu \mathrm{m} /$ [SIII] $33.48 \mu \mathrm{m}$ (top) and [OIV] $25.89 \mu \mathrm{m} /$ [NeII] $12.81 \mu \mathrm{m}$ (middle). Only those lines detected at more than a $3 \sigma$ level are included in the above mapping. Line ratios are corrected for foreground extinction based on the ratio between $10 \mu \mathrm{m}$ and $14 \mu \mathrm{m}$ continuum fluxes. Bottom: Schematic diagram of key features in the CMZ. The grey circles represent the location of 107 YSO candidates in the Spitzer/IRS survey. [A COLOR VERSION IS AVAILABLE ONLINE.] 


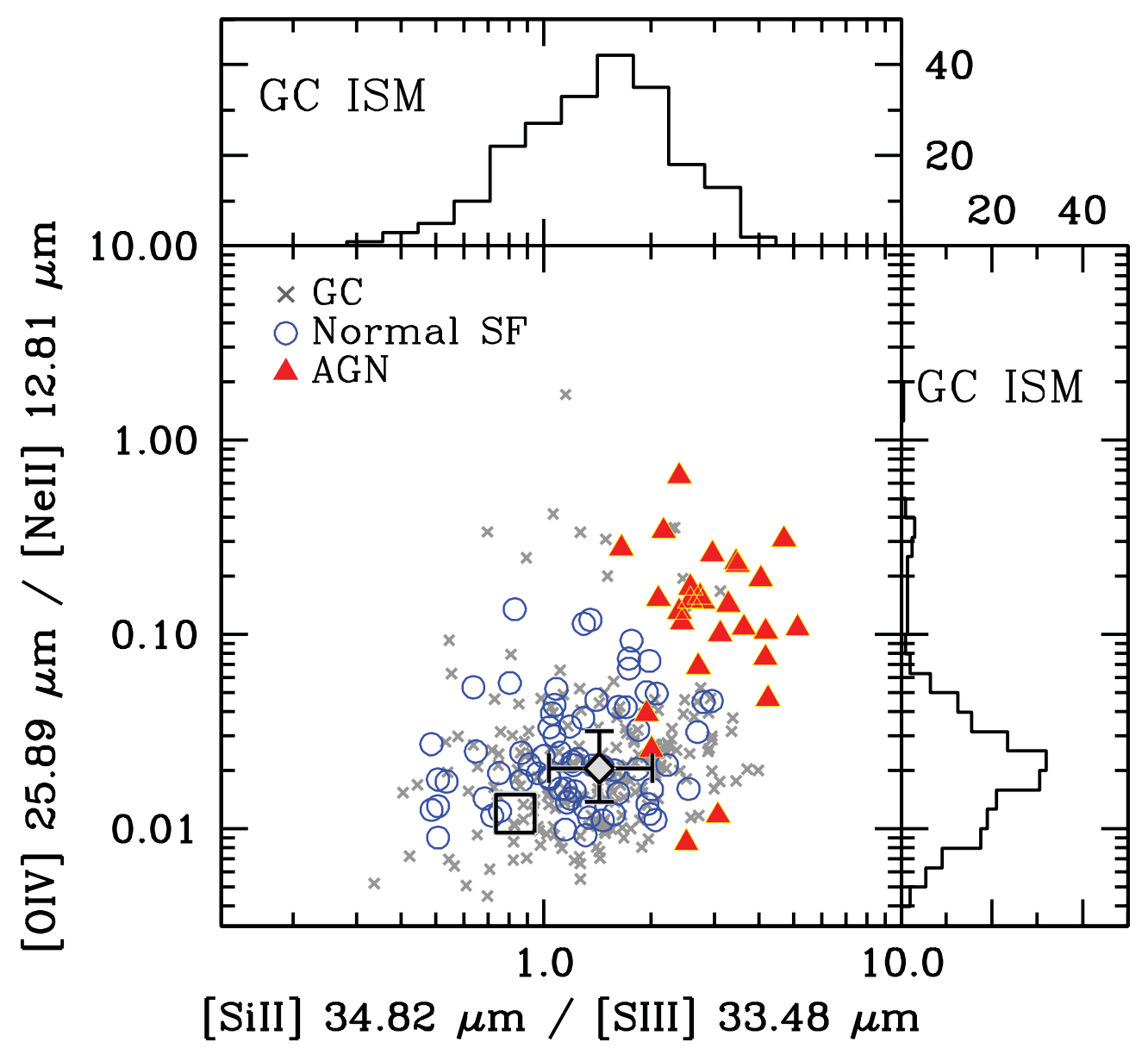

Figure 3. Line-ratio diagram with [SiII] $34.82 \mu \mathrm{m} /$ [SIII] $33.48 \mu \mathrm{m}$ versus [OIV] $25.89 \mu \mathrm{m}$ / [NeII] $12.81 \mu \mathrm{m}$. Grey points represent individual Galactic center (GC) spectra, and their number distributions are shown on each axis. Filled diamond point shows the median line ratio in the GC, with interquartile ranges of line ratios indicated by error bars. Open box is the line flux ratio from the coadded GC spectrum. Normal star-forming regions (both nuclear and extra-nuclear) and AGNs in the SINGS study (Dale et al. 2009) are displayed as open blue circles and filled red triangles, respectively. [A COLOR VERSion is AVAILABLE ONLINE.]

order of magnitude larger than the area covered by a slit entrance of either SH or LH. Mean line ratios are shown in each pixel, which includes two background IRS pointings on average. Our mapping is superior to previous survey results in terms of the total area covered in the GC.

Empirical evidence suggests that some mid-IR line ratios are useful diagnostic tools for discriminating between normal star-forming regions and AGNs (e.g., Lutz et al. 1998; Sturm et al. 2006; Dale et al. 2009). Figure 3 displays one set of mid-IR line diagnostics to separate AGNs from normal star-forming regions, which plots [SiII] $34.82 \mu \mathrm{m} /$ [SIII] $33.48 \mu \mathrm{m}$ versus [OIV] $25.89 \mu \mathrm{m} /$ [NeII] $12.81 \mu \mathrm{m}$. The red filled triangles are AGNs, and blue open circles are normal star-forming regions in the SINGS sample (Dale et al. 2009). Grey cross points represent our measurements from individual GC spectra. We only included emission lines with more than $3 \sigma$ detections. Number distributions of ionic line ratios are shown on each axis. 
As seen in Figure 3, almost all GC data points have ratios agreeing with the line ratios observed in normal star-forming regions in nearby galaxies. The median value from the individual spectra of the CMZ is indicated by a grey diamond point, and the error bars represent the interquartile ranges of distributions of individual GC spectra. The line ratio for the coadded GC spectrum, which is displayed as an open box, also indicates that the emission-line properties of the $\mathrm{CMZ}$ are similar to those of extragalactic star-forming regions.

We conclude that our observations of mid-IR line emission in the central $210 \mathrm{pc} \times 60 \mathrm{pc}$ of the Milky Way Galaxy show no evidence of excitation by a power-law continuum by an AGN. About 3\% of GC points fall in the AGN territory in Figure 3. However, they are located at high Galactic latitudes (see Figure 2), and are likely outliers, such as those excited by very hot sources (e.g., Wolf-Rayet stars, planetary nebulae, or X-ray binaries), rather than belonging to an AGN-like trend. Our results agree with those of Simpson et al. (2007) and Contini (2009), who conclude based on a smaller Spitzer data set than in this study that the mid-IR line ratios can be explained by photoionization by hot stars, combined with shocks.

\section{Acknowledgements}

D. A. acknowledges support by National Research Foundation of Korea (2010-0025122).

\section{References}

An, D., Ramírez, S. V., \& Sellgren, K. 2013, ApJS 206, 20

An, D., Ramírez, S. V., Sellgren, K., et al. 2009, ApJ Lett. 702, L128

An, D., Ramírez, S. V., Sellgren, K., et al. 2011, ApJ 736, 133

Contini, M. 2009, MNRAS 399, 1175

Dale, D. A., et al. 2009, ApJ 693, 1821

Kennicutt, R. C., Jr., Armus, L., Bendo, G., et al. 2003, PASP 115, 928

Lutz, D., Kunze, D., Spoon, H. W. W., \& Thornley, M. D. 1998, A\&A Lett. 333, L75

Morris, M. \& Serabyn, E. 1996, ARAA 34, 645

Simpson, J. P., Colgan, S. W. J., Cotera, A. S., et al. 2007, ApJ 670, 1115

Sturm, E., Rupke, D., Contursi, A., et al. 2006, ApJ Lett. 653, L13 